\title{
Evolución de una hernia perineal canina resuelta por transposición del músculo semitendinoso
}

\author{
González, R. ; Britez, C. ; Bazán, Y.2.; Caballero, M.J. 2; Portillo, C. ${ }^{3}$ \\ ${ }^{1}$ Cátedra Técnica Operatoria, ${ }^{2}$ Depto.Post Grado, ${ }^{3}$ Tesista de Grado, \\ Facultad Cs. Veterinarias, Univ. Nac. de Asunción, San Lorenzo, Paraguay. \\ E-mail: rgonzalez@vet.una.py
}

\begin{abstract}
Resumen
González, R.; Britez, C.; Bazán, Y.; Caballero, M.J.; Portillo, C.: Evolución de una hernia perineal canina resuelta por transposición del músculo semitendinoso. Rev. Vet. 31: 2, 160-164, 2020. El objetivo del trabajo fue determinar la evolución clínica de un caso de hernia perineal en un canino mestizo, macho de 11 años, resuelto por la técnica de transposición del músculo semitendinoso. Se realizaron evaluaciones clínicas post operatorias, evaluando presencia/ausencia de recidivas del abultamiento y además presencia/ausencia de patologías de la cicatrización como úlceras, fístulas, cicatrices hipertróficas, queloides, seromas y también dehiscencia y tumefacción. Los resultados obtenidos a los 30 días de evaluación arrojaron ausencia de dolor, inflamación, dehiscencia, úlceras, fístulas, seromas, cicatrices hipertróficas, queloides y recidivas del abultamiento, todos ellos considerados signos favorables dentro del presente estudio. La técnica operatoria resultó muy sencilla, el anclaje de los puntos fue fácil, sin ninguna dificultad. La manipulación tisular, a pesar de la incisión bastante amplia, no generó ninguna complicación más allá de la hemorragia, resultando una técnica bastante efectiva.
\end{abstract}

Palabras clave: canino, hernia perineal, transposición del músculo semitendinoso.

\begin{abstract}
González, R.; Britez, C.; Bazán, Y.; Caballero, M.J.; Portillo, C.: Evolution of a canine perineal hernia resolved by transposition of semitendinous muscle. Rev. Vet. 31: 2, 160-164, 2020. The objective of the present research was to determinate the clinical evolution of a case of perineal hernia in an 11-year-old canine half breed, solved by the technique of the transposition of the semitendinous muscle. Post-operative clinical evaluations were made, evaluating presence / absence of pathologies of healing such as ulcers, fistulas, hypertrophic scars, keloids, seromas and tumefactions. The results obtained at 30 days of evaluation, showed no pain, inflammation, ulcer, fistula, seroma, hy pertrophic scar, keloid and recurrent bulging, all considered favorable signs within the present study. The tissue manipulation, due to the fairly wide incision, did not generate complication beyond the hemorrhage, being an effective technique.
\end{abstract}

Key words: canine, perineal hernia, transposition of the semitendinous muscle.

\section{INTRODUCCIÓN}

La hernia perineal es el resultado de la falla del diafragma pélvico muscular para soportar a la pared del recto, el cual se estira y desvía. El contenido pélvico, y en ocasiones el abdominal, puede protruir entre el diafragma pélvico y el recto. Así, se produce una tumefacción subcutánea ventrolateral al ano y -en las hernias bilaterales- también es factible observar la proyección caudal del ano ${ }^{12}$.

El contenido de la hernia está rodeado por una fina capa de fascia perineal (saco herniario), tejido subcutáneo y piel. El saco puede contener grasa pélvica o retroperitoneal, líquido seroso, un recto desviado o dilatado, un divertículo rectal, la próstata, la vejiga de la orina o el intestino delgado. La retroflexión vesical ocurre en aproximadamente el $20 \%$ de los pacientes.

Las hernias perineales son frecuentes en perros y raras en gatos. Ocurren casi exclusivamente en perros machos enteros (93\%). En perras, a menudo están relacionadas con traumatismos. La mayoría de las hernias perineales ocurre en perros de más de 5 años de edad. La edad media de presentación es de aproximadamente 10 años tanto en perros como en gatos ${ }^{1,7}$.

La herniación perineal puede ser unilateral o bilateral. Algunos investigadores comunicaron una mayor incidencia de hernia perineal sobre el lado derecho, pero el criterio empleado para determinar unilateral vs bilateral e izquierda vs derecha es subjetivo ${ }^{1,4,8}$. 
Los signos clínicos incluyen el abultamiento del periné, generalmente reducible, a uno o ambos lados del ano, según se trate de una hernia uni- o bilateral. Se puede hallar también: estreñimiento, tenesmo y disquecia, que en ocasiones alternan con diarrea o incontinencia fecal. Si la vejiga forma parte del contenido de la hernia y se encuentra en retroflexión, el cuadro clínico puede agravarse al ser imposible la micción, llegándose a producir una azoemia post-renal ${ }^{2,11}$.

La hernia perineal puede resolverse quirúrgicamente mediante múltiples técnicas. La elección de la más adecuada para cada caso depende principalmente del estado de la musculatura del periné (tamaño y localización del anillo, mayor o menor grado de atrofia muscular), el tipo de contenido herniario (intestino, vejiga) y la experiencia del cirujano con cada una de las técnicas. Entre las técnicas quirúrgicas indicadas para la resolución de la hernia perineal se encuentran: herniorrafia simple, elevación o transposición del músculo obturador interno, transposición del músculo glúteo superficial, transposición del músculo semitendinoso, colocación de prótesis tipo malla, cistopexia, deferentopexia y colopexia Amsterdam ${ }^{5,11}$

La recurrencia de la herniación perineal canina luego de la herniorrafia tradicional puede alcanzar hasta el $46 \%$. Se han comunicado tasas de recurrencia de apenas $5 \%$ para la técnica de herniorrafia con transposición del músculo obturador interno. Cuando la técnica falla o se considera con escasas posibilidades de éxito, se recomienda optar por algunas de las siguientes metodologías: transposición del músculo semitendinoso para la reconstrucción perineal o colopexia (o cistopexia) mediante fijación del conducto deferente para prevenir la herniación de estructuras importantes.

La transposición del músculo semitendinoso es de particular utilidad para las reconstrucciones en las cuales la zona ventral del perineo está muy afectada, como es el caso de algunas hernias perineales bilaterales. Para la herniación perineal unilateral, el músculo semitendinoso contralateral se recomienda para la reconstrucción del diafragma pélvico ${ }^{1,9}$.

La presente investigación tuvo como objetivo determinar la evolución clínica de un caso de hernia perineal unilateral en un canino, resuelta por medio de la técnica de transposición del músculo semitendinoso.

\section{MATERIAL Y MÉTODOS}

Para el estudio se utilizó un canino macho de sexo entero, raza mestiza, 11 años de edad y $4,3 \mathrm{~kg}$ de peso, con diagnóstico clínico de hernia perineal con hiperplasia prostática, de 6 meses de evolución. Según lo manifestado por el propietario, aparte del abultamiento, el paciente reportaba tenesmo y estreñimiento.

Durante el examen clínico, la palpación indicó la presencia de un abultamiento indoloro reducible, sin aumento de temperatura local. Se realizó una palpación rectal con el dedo índice provisto de un guante lubricado, no detectándose saculaciones ni materia fecal impactada, por lo cual se llegó al diagnóstico presuntivo de hernia perineal.

Para detectar alteraciones relacionadas con la próstata, se solicitó una ecografía cuyo resultado reportó una próstata de tamaño aumentado $(1,8 \mathrm{~cm}$ de diámetro), localización abdominal, parénquima heterogéneo, bordes irregulares y zonas anecoicas en su interior, asumiendo el diagnóstico imagenológico de hiperplasia prostática y hernia perineal con contenido intestinal. Realizados los estudios pre-quirúrgicos de rutina, y los exámenes complementarios, se procedió a la programación de la cirugía.

El campo operatorio fue adecuadamente preparado e inicialmente el paciente fue colocado en decúbito dorsal para proceder a la orquiectomía pre-escrotal de rutina, a testículo y cordón cubierto, previo embrocado con solución de clorhexidina al $0,5 \%$ mediante aspersión, para el tratamiento de la patología prostática. Finalizada la orquiectomía, se posicionó al paciente en decúbito esternal, con los miembros posteriores suspendidos, se preparó el campo de la manera ya descrita, colocando los paños de campo correspondientes y realizando una sutura en jareta del ano.

Con el perro en la posición perineal, se realizó una incisión cutánea en la piel perineal desde la base del rabo hasta el rafe medio en ventral, similar a la reparación tradicional, con la transposición del músculo obturador interno, y se la continuó a través de la línea media hacia la tuberosidad isquiática, donde se curvó y dirigió distalmente sobre la zona caudal del miembro pélvico, finalizando en el área caudo-medial de la transición entre la rodilla y caña de la pierna.

Los contenidos herniarios se expusieron y redujeron, como en otras técnicas de herniorrafia, antes de aislar el músculo semitendinoso. Los tejidos subcutáneos sobre dicho músculo se seccionaron para su exposición. El músculo semitendinoso se aisló en forma roma desde las estructuras circundantes (Figura 1), con la cautela de no lesionar el pedículo vascular proximal (arteria y vena glúteas caudales).

El músculo semitendinoso se transeptó lo más distalmente posible, cerca de la rodilla, y se lo aisló para movilizarlo hasta la región perineal. Empleando poliamida, la porción transeptada del músculo semitendinoso se suturó sobre el defecto de la hernia (Figura 2).

La piel se suturó mediante puntos simples entrecortados (Figura 3). El sitio quirúrgico se lavó con solución salina estéril y se mantuvo limpio durante el postoperatorio inmediato. La regulación de la dieta facilitó una defecación regular y evitó excesivos esfuerzos, consistiendo en alimentación líquida o pastosa, fácilmente digestible.

Durante el post-operatorio el paciente fue administrado con cefalexina oral a dosis de $22 \mathrm{mg} / \mathrm{kg}$ cada 12 horas durante 10 días, ketorolaco por vía oral a dosis de $2 \mathrm{mg} / \mathrm{kg}$ cada 24 horas durante 3 días y tramadol vía oral $2 \mathrm{mg} / \mathrm{kg}$ cada 6 horas durante 2 días.

Para evaluar el resultado de la técnica, se estudió la evolución clínica a los 2, 8, 15 y 30 días (Tabla 1), 
Tabla 1. Signos clínicos evaluados en el paciente en estudio.

\begin{tabular}{cccccc}
\hline evaluación & úlceras & fístulas & seromas & cicatriz & queloides \\
\hline día 2 & ausente & ausente & presente & ausente & ausente \\
día 8 & ausente & ausente & ausente & ausente & ausente \\
día 15 & ausente & ausente & ausente & ausente & ausente \\
día 30 & ausente & ausente & ausente & ausente & ausente \\
\hline
\end{tabular}

*hipertrófica

investigando presencia/ausencia de recidivas del abultamiento, y presencia/ausencia de patologías de la cicatrización: úlceras, dehiscencia, fístulas, cicatriz hipertrófica, queloides y seromas.

\section{RESULTADOS Y DISCUSIÓN}

La primera evaluación post-operatoria fue realizada a las 48 h de finalizado el procedimiento (Figura 4). Se constató aumento de la sensibilidad a la palpación de la región (tumefacción) y secreción de tipo serosasanguinolenta que emanaba por la herida (seroma), lo cual se consideró normal dentro del proceso fisiológico de la cicatrización, debido a la manipulación tisular, no observándose úlceras, dehiscencia, fistulas, cicatrices hipertróficas ni queloides.

Todos los signos encontrados (tumefacción local, edema y dolor a la palpacióny presión) son coincidentes con afirmaciones de otros investigadores ${ }^{13}$. El rubor se debe al gran aumento de sangre en la zona inflamada. El tumor o hinchazón resulta del aumento de la sangre y de la presencia adicional de sustancias que exudan de los vasos sanguíneos en los tejidos vecinos. El calor también resulta del aumento de la corriente sanguínea, que eleva la temperatura de los órganos internos hacia la periferia. El dolor es atribuido a la elevación de la presión sobre las terminales nerviosas.

En el día 8 la palpación de la zona presentó sensibilidad con una ligera inflamación, sin dehiscencia ni fístula, ausencia de seroma y otras patologías de la cicatrización (Figura 5). Los fenómenos inflamatorios disminuyeron en intensidad. La fase fibroblástica de la cicatrización suele durar entre 2 a 4 semanas dependiendo de la herida, y a medida que el contenido de colágeno aumenta, disminuyen las glicoproteínas y mucopolisacáridos, así como la síntesis de fibroblastos. Los capilares comienzan a regresar y el colágeno sintetizado disminuye para equilibrar finalmente la proporción de colágeno destruido ${ }^{12}$.

A los 15 días fueron retirados los puntos de sutura. Tanto en el día 15 como en el 30 , ya no se percibió aumento de sensibilidad, inflamación, fístulas, seromas, ni ninguna otra patología de la cicatrización (Figura 6). La técnica de transposición del músculo semitendinoso puede usarse en animales con musculatura perineal muy debilitada o atrofiada, así como también en hernias de presentación bilateral. En casos de hernia unilateral, para su reconstrucción se utiliza el músculo semitendinoso contra-lateral.
En un estudio se evaluó la misma técnica estudiada en este trabajo, donde los perros intervenidos no presentaron problemas locomotores o de movimiento articular en el miembro operado ${ }^{9}$; por otro lado, se observó que el músculo transpuesto se mantuvo contráctil por 90 días, aunque posteriormente sufrió un proceso atrófico.

Algunas de las mayores complicaciones observadas al aplicar esta técnica fueron la acumulación de secreciones o la dehiscencia de los puntos, que pueden ser reducidas mediante el uso de drenajes. En el paciente utilizado para el estudio no se observó ningún tipo de problema locomotor.

Otros investigadores realizaron un estudio retrospectivo de los casos de hernia perineal, los cuales representaron el $0,96 \%$ de la población ${ }^{10}$. Los machos

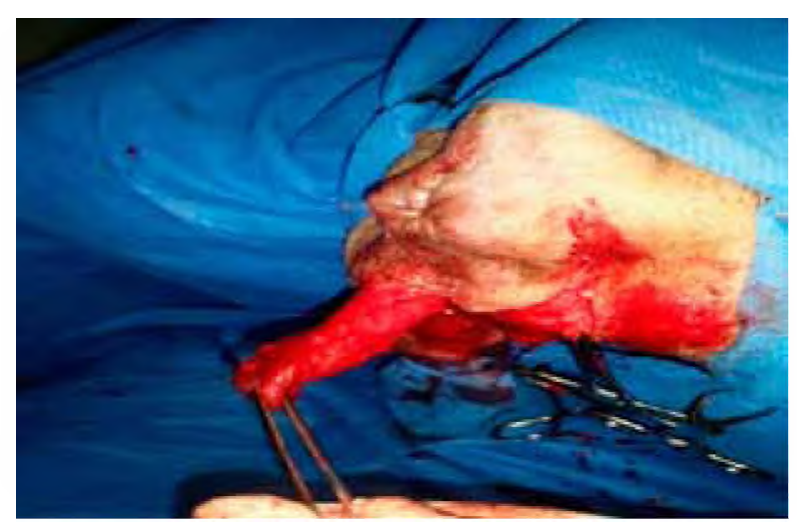

Figura 1. Sección del músculo semitendinoso.

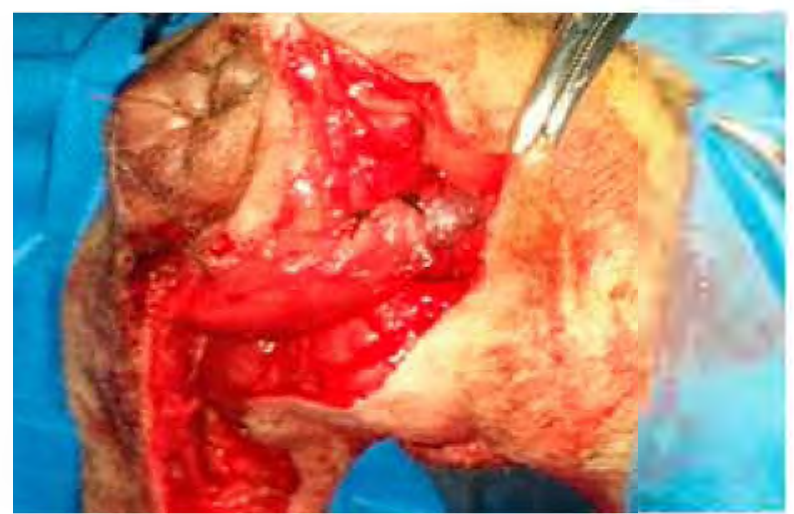

Figura 2. Sutura del músculo semitendinoso sobre el defecto de la hernia.

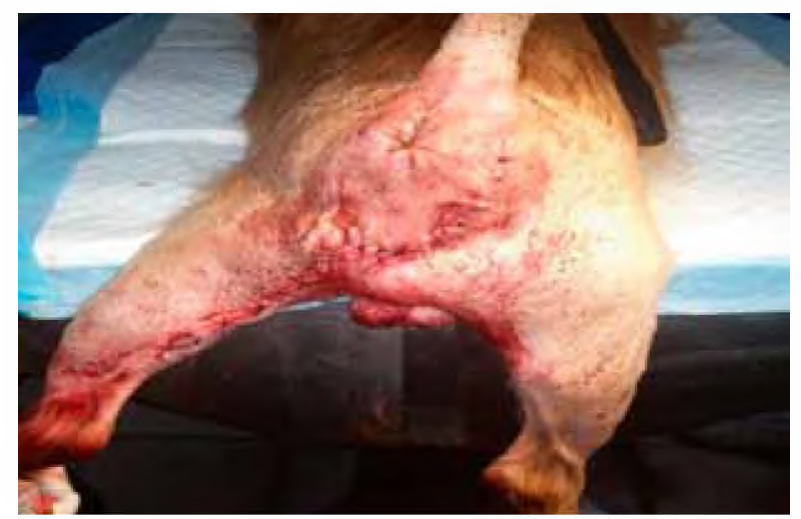

Figura 3. Cirugía finalizada. Nótese la extensa incisión realizada. 


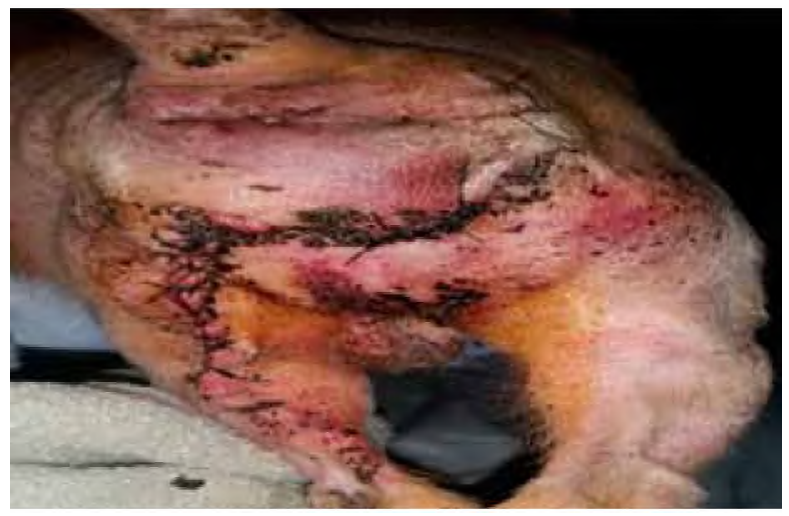

Figura 4. Evolución de 48 horas.

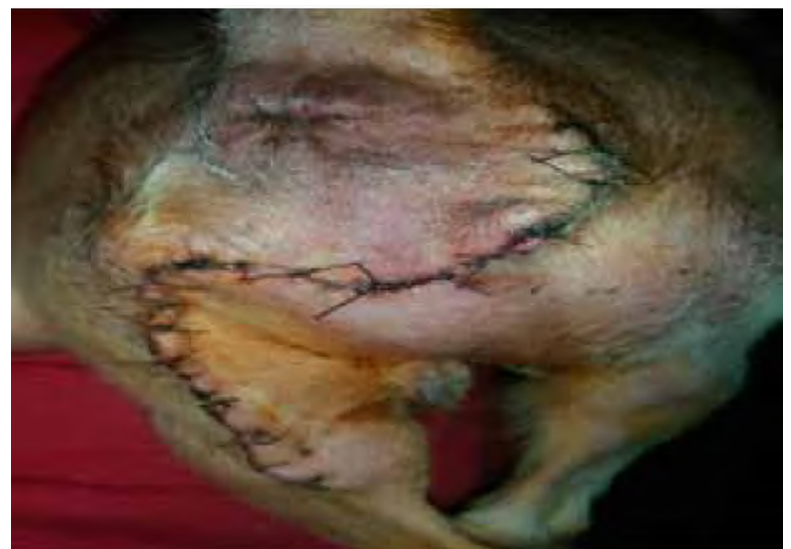

Figura 5. Evolución de 8 días.

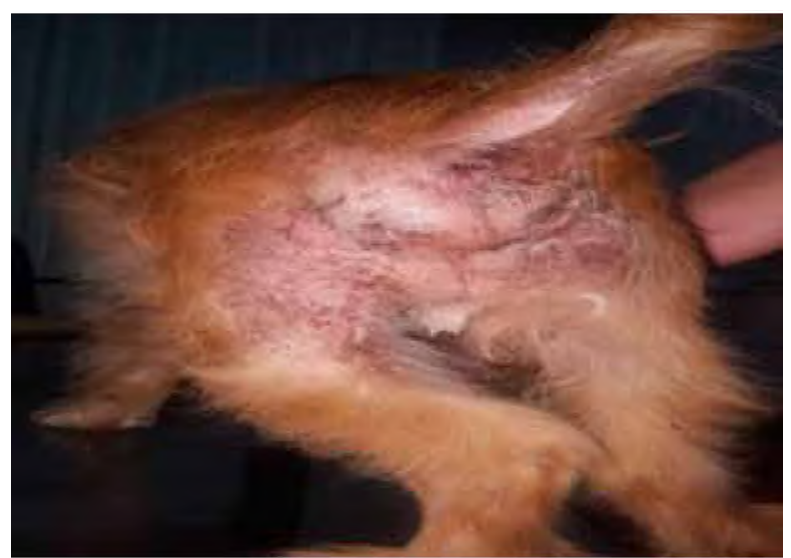

Figura 6. Evolución de 30 días.

constituyeron un $98,2 \%$ y las hembras $1,2 \%$. Los pacientes tenían una edad media de $8,69 \pm 2,19$ años y un peso de $16,4 \pm 11,44 \mathrm{~kg}$ (media \pm desviación típica). Se encontraron 18 hernias perineales bilaterales, 40 unilaterales derechas y 20 unilaterales izquierdas, con 96 hernias perineales. Fueron intervenidos 67 pacientes, sumando 80 hernias perineales operadas. El 13,6\% de los casos presentó retroflexión de vejiga y el $21 \%$ afectación de la próstata.

Tales daños fueron resueltos por medio de la técnica de transposición del obturador interno ${ }^{10}$, siendo parcialmente coincidentes con los presentados en el paciente estudiado en esta investigación, el cual ostentaba una hernia unilateral derecha con afectación de la próstata.
Entre los años 2007 y 2012 se realizó un estudio con el objetivo de determinar la frecuencia de perros afectados por hernias perianales ${ }^{3}$. Los resultados obtenidos indicaron que los caninos mayormente afectados fueron machos ( $92,5 \%$ de un total de 80 animales), mayores de 5 años $(87,5 \%)$, alimentados con comida casera y balanceado. Estos resultados coinciden con lo reportado en el paciente estudiado ya que en la anamnesis el propietario manifestó alimentar a su mascota con comida casera y balanceado.

Según otro estudio, la tasa de recidiva de la enfermedad depende de diversos factores ${ }^{10}$, señalando que la inexperiencia del cirujano podría ser un factor clave, con tasas de recidiva muchísimo mayores para cirujanos inexpertos.

La técnica quirúrgica también puede considerarse un factor clave en la tasa de recidiva, siendo muy variable según el procedimiento empleado. Para la transposición del músculo obturador interno hay referencias de una recurrencia del $2,4 \%$ al $19 \%$. No se encontraron estudios sobre la recurrencia empleando la técnica de transposición del músculo semitendinoso. La anatómica recoge en este estudio tasas de recurrencia mucho más altas. Así, en otro estudio en el que también se usaron ambas técnicas, se describe una recidiva del $8 \%$ y dicho porcentaje se ciñe exclusivamente a la anatómica.

En cualquier caso, las tasas de recidiva utilizando técnicas de colgajos musculares son muy bajas; está claro que se requiere un aprendizaje previo para reducir las recidivas por inexperiencia del cirujano, pero debe considerarse una técnica fiable y segura para la herniorrafia perineal.

Asimismo, no se considera necesario adicionar una malla de polipropileno a la técnica, ya que tanto estos resultados como los encontrados en la bibliografía, no muestran disminución significativa de las recidivas, además de que la colocación de la malla supone un costo extra, que no parece necesario y prolonga el tiempo de cirugía ${ }^{6}$.

En cuanto a la valoración del método y la técnica operatoria, debido a la gran manipulación tisular en la región del procedimiento quirúrgico, se pueden evidenciar muchos fenómenos relacionados a la inflamación. La hemorragia fue moderada aplicando dicha técnica y el cierre del defecto herniario se produjo de manera completa, no existiendo tensión excesiva en la rafia muscular.

Según los resultados obtenidos luego de 30 días de evaluación, se concluye que hubo ausencia de seromas, úlceras, fistulas y recidivas del abultamiento. Tampoco fueron observadas patologías cicatrizales dentro de los tiempos esperados.

Se recomienda la realización de estudios similares a fin de tener mayor base de datos y respaldo a la hora de implementar esta técnica quirúrgica, ya sea en caninos u otras especies animales, utilizando distintas técnicas, como transposición del obturador interno, cistopexia, deferentopexia, o colopexia. 


\section{REFERENCIAS}

1. Bojrab J, Ellison G, Slocum B. 2000. Técnicas actuales en cirugía de pequeños animales, 4ta ed., Edit. Intermédica, Buenos Aires, $1276 \mathrm{p}$.

2. Couto CG, Nelson RW. 2000. Medicina interna de animales pequeños, 2 da ed., Edit. Intermédica, Buenos Aires, $1467 \mathrm{p}$.

3. Cuenca JA. 2014. Frecuencia de hernias perineales en caninos que concurrieron al Hospital de la Facultad de Ciencias Veterinarias de la Universidad Nacional de Asunción, en los años 2007 al 2012. Tesis de Doctorado, FCV, UNA.

4. Dyce KM, Sack WO, Wensing CJ. 2012. Anatomia veterinaria, 4ta. ed., Edit. Manual Moderno, Mexico, 833 p.

5. Ettinger SJ. 2007. Tratado de medicina interna veterinaria: enfermedades del perro y el gato, 6ta ed., Edit. Elsevier, Amsterdam, $912 \mathrm{p}$.

6. Fernandez MA. 2016. Hernia perineal canina: reconstrucción del diafragma pélvico con malla de polipropileno a través de doble abordaje. Tesis Doctoral, Universidad Complutense de Madrid, $69 \mathrm{p}$.
7. Fossum T. 2009. Cirugía en pequeños animales, 3ra ed., Editorial Elsevier, Barcelona, $1.610 \mathrm{p}$.

8. Konig HE, Liebich H. 2008. Anatomía de los animales domésticos, 2da ed., Edit. Panamericana, Buenos Aires, $400 \mathrm{p}$.

9. Möller R. 2015. Evaluación y caracterización de las mallas de polietileno de alta densidad para su uso como implantes en cirugía veterinaria (on line). https://buleria. unileon.es/bitstream/handle/10612/4180/tesis_504cd9.PDF.

10. Ramírez A, Pastor N, Durán ME, Gutiérrez A, Ezquerra L. 2015. Hernia perineal en el perro, estudio de prevalencia de 81 casos. http://eds.b.ebscohost.com/eds /pdf. viewer $/$ pdfviewer?vid $=9 \&$ sid $=5913 f 3 f 8-5$ cbc- 452 c-bf $33-$ $8 \mathrm{e} 32 \mathrm{ea} 353 \mathrm{f} 85 \%$

11. Rodriguez GJ, Graus MJ, Martinez SM. 2005. Cirugia en la clínica de pequeños animales. la parte posterior. Edit. Servet, Zaragoza, España, 296 p.

12. Slatter D. 2006. Tratado de cirugía en pequeños animales, 3ra ed., Intermédica, Buenos Aires, 895 p.

13. Smith HA, Jones TC. 1987. Patologia veterinaria, 2da. edición, Ed. Hispano-Americana, México, $1023 \mathrm{p}$

\section{Revista Veterinaria obtuvo el máximo nivel de categorización del CAICYT-CONICET}

Tras el pertinente proceso de evaluación según criterios de calidad editorial, en setiembre de 2005 CAICYT-CONICET ha clasificado a nuestra publicación con Categoría 1 (nivel superior de excelencia), con lo cual pasa a integrar el Catálogo Latindex (folio 14022). La Dirección de Revista veterinaria agradece a quienes colaboraron para obtener tan importante distinción.

Ver: http://www.latindex.unam.mx/busquedas/catalogotitulo.html 